\title{
Case Studies of a Borehole Deployable Robot for Limestone Mine Profiling and Mapping
}

\author{
A. Morris \\ D. Kurth \\ Robotics Institute: Field Robotics Center \\ Carnegie Mellon University \\ Pittsburgh, PA 15213 \\ \{acmorr, dekurth, dhuber, sthayer\}@cs.cmu.edu
}

D. Huber

${ }^{\ddagger}$ C. Whittaker

S. Thayer

\author{
${ }^{\ddagger}$ Workhorse Technologies, LLC. \\ 484 West $7^{\text {th }}$ Avenue \\ Homestead, PA 15120 \\ chuck@workhorsetech.com
}

\begin{abstract}
Inherent dangers in mining operations motivate the use of robotic technology for addressing hazardous situations that prevent human access. In the context of this case study, we examine the application of a robotic tool for map verification and void profiling in abandoned limestone mines for analysis of cavity extent. To achieve this end, our device enables remote, highly accurate measurements of the subterranean voids to be acquired. In this paper we discuss the design of the robotic tool, demonstrate its application in void assessment for prevention and response to subsidence, and present results from a case study performed in the limestone mines of Kansas City, Kansas.
\end{abstract}

\section{$1 \quad$ Introduction}

The existence of subterranean void spaces, such as the cavities created by mining, is a hazard to active mining operations and a constant threat to surface developments. When abandoned, these underground spaces can accumulate tremendous quantities of water and threaten to flood encroaching active mines (as occurred in Quecreek, PA on July 2002 [1]) or subside to form sinkholes on the surface [2]. Although both hazards have devastating consequences if left unchecked, subsidence is the major concern for aboveground development since it degrades the structural integrity of the overlaying land. With an estimated 500,000 abandoned mines in the United States, subsidence is a formidable problem for community and city infrastructure [3].

Kansas City, Kansas, is one particular area plagued with problems of subsidence. Underneath the city reside massive limestone mines that have been sealed and abandoned for several years. During the operation of these mines, the "room-and-pillar" mining techniques utilized for mineral extraction left mined areas susceptible to ceiling collapse. Older mining techniques removed excessive amounts of limestone. Over time, exposed and structurally weak shale layers yielded persistent degradation in roof rock support and produced ceiling collapses. Referred to as "domeouts," these collapses typically generated void volumes in the range of 3,800 to 35,000 cubic meters. If left unattended, these cavities propagate to the surface forming sinkholes that risk surface structures and prohibit future land development.

In response to the situation, a remediation technique was developed to restore the structural integrity of land situated above a domeout. Utilizing flyash (a waste product from coal fired electric generating plants) and water, a cement-like slurry is created. This slurry is poured into domeouts through boreholes (Figure 1). Once completely backfilled and cured, the domeout is considered stable [4]; however, a successful backfill operation is only achieved with the proper preparation and mine information. The underlying challenges posed by backfilling are (1) obtaining reliable void dimensions and volume estimations, (2) verification and identification of domeout location on the mine map, and (3) registration of the mine map in a surface coordinate frame.

To address the backfilling challenges, we developed a robotic tool that is capable of reaching a domeout via borehole access (the same hole used for pouring the coal flyash slurry), acquiring the measurements necessary for void analysis, and relaying this information to the surface. To reach the mine cavity, deployment and sensing schemes were required to descend the borehole, identify the mine breach, and maintain a sense of orientation throughout the process. For measuring the cavity, sensing, actuation, and mapping methods were required to scan the whole of the void. In this paper, we explain the design; demonstrate the application; and discuss the results from a case study that involved assessment of a prior backfilling operation in the limestone mines of Kansas City. 


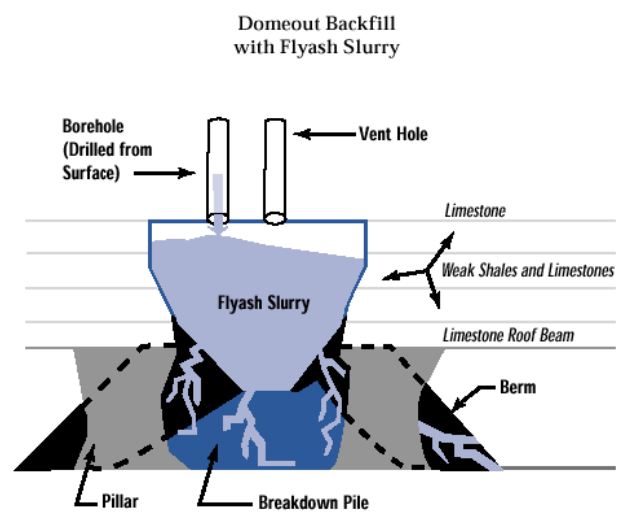

Figure 1: The process of backfilling a domeout with coal flyash (courtesy of [4])

\section{$2 \quad$ Related Methods}

Current methods for underground void detection include non-intrusive techniques such as ground-penetrating radar (GPR) and microgravity as well as direct methods such as borehole-deployed cameras and human surveying. With GPR, a surveyor acquires information about subsurface structures by analyzing the response of high frequency electromagnetic waves propagated through the ground. These waves are reflected back to the surface when they encounter a sharp change in the electrical properties of underground matter, and this response can be analyzed to identify voids or other features of interest. Due to attenuation of the EM waves, GPR is typically limited to depths of no more than $30 \mathrm{~m}$, with scan resolution worsening proportionate to scan depth, making this method appropriate chiefly for shallow investigations $[5,6]$.

Microgravity techniques require careful measurement of variations in the Earth's gravitational field, which can be used to infer void presence, depth, and shape. Again, while non-intrusive and non-destructive like GPR, this method is viable only to depths on the order of tens of meters [7].

When human access is possible, inspectors may explore and evaluate mine remnants, but this may pose great personal risk to the surveyor, particularly when surveying around domeouts or other collapses. When conditions preclude human access, remote cavity detection can be obtained by borehole drilling. A hole is bored from the surface to an assumed mine cavity at a known depth and map information is inferred by the presence or absence of ground materials. To augment borehole findings, cameras may be deployed to visually affirm the presence of void space in situations where a cavity is found $[8,9]$. The caveat with borehole and camera techniques is that they provide limited information. Qualitative range data (distance measurements) cannot be extracted and void extent is not explicitly measurable. As a result, boreholes must be densely distributed across the encompassing mine area, requiring a substantial investment of time, money, and resources.

\section{$3 \quad$ Platform Design}

The robotic tool we developed for remote subterranean void analysis has several operational advantages over past techniques. Nicknamed Ferret, it establishes a physical presence in a mine cavity enabling a "first hand" perspective of the void (unlike non-intrusive methods that require information to be inferred). This physical presence is attained without human presence in or around the mine, which removes the risks faced by surveyors in subterranean inspections. In addition, Ferret provides quantitative information on cavity extent that is difficult or impossible to obtain using borehole camera systems. In the following sections, we describe the mechanical, electrical, and software systems that compose the Ferret and empower it to retrieve cavity data in a rugged and unforgiving environment.
(A)

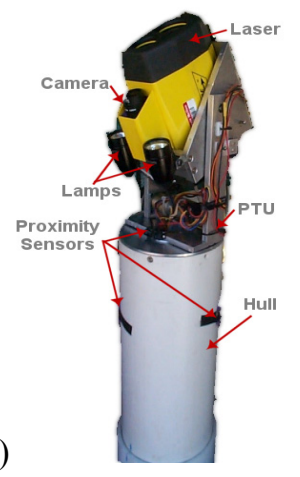

(B)

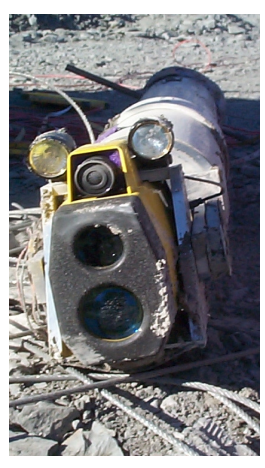

Figure 2: Views of Ferret. (A) The side-view layout and (B) shows Ferret after $2^{\text {nd }}$ deployment.

\subsection{Mechanical System}

The Ferret device is a relatively simple mechanism that provides extreme robustness and reliability in harsh environmental conditions. Ferret's primary mapping sensor is a single-point, long-range, low-reflectivity laser that performs measurements on the cavity enclosure. To obtain a semi-spherical scan of the void, the laser is actuated on a pan and tilt unit (PTU) with an effective motion range of $360^{\circ}$ in the horizontal plane and $150^{\circ}$ in the vertical plane. The PTU is driven with two $12 \mathrm{Vdc}$ motors that independently control each degree of 
freedom. The motors are highly geared to minimize backlash and maximize the accuracy of angular displacement.

Above the laser and PTU is a cylindrical hull constructed of 6" PVC pipe that encapsulates sensitive computing and sensing modules. At the upper end of the hull, a thick aluminum plate provides an interface connector and support bar. The interface connection enables a communications and power linkage between Ferret and the surface control station. The support bar connects Ferret to a 3/16 in. diameter steel cable that suspends the device from the surface during an operation. This cable is drawn through a motor-driven winch and pulley system for deployment and retrieval.

\subsection{Electrical and Sensing System}

As previously mentioned, the primary mapping sensor utilized on Ferret is a surveying laser manufactured by the Atlanta Optics Company ${ }^{\mathrm{TM}}$. The laser is capable of range measurements in excess of $50 \mathrm{~m}$ on materials found in mines like coal, limestone, shale, and bedrock. The laser is equipped with a magnetic compass and a two-axis tilt sensor to detect pitch and roll. A low-light camera is mounted alongside the laser with two small lamps to allow visualization of Ferret's progress. Furthermore, the camera provides visual feedback to assist human operators in teleoperation of Ferret.

Just above the laser, an analog IR proximity sensor is mounted to the PTU frame to provide distance measurements at small ranges $(12 \mathrm{~cm})$ perpendicular to Ferret's hull. The limited measuring range serves as a breach detection system that signals to the command station when the cavity opens beyond the sensors perceptive range. Two other digital IR sensors are mount along the hull's side to assist in determination of device depth.

Ferret is also equipped with encoders, magnetic homing switches, and a hull-mounted compass. These sensors provide Ferret and its laser orientation information relative to a global bearing (i.e. the Earth's magnetic field) as well as means of control.

Ferret's central computing is located within the hull and consists of an embedded $18.432 \mathrm{MHz}$ Rabbit 2000 microprocessor. This processor is responsible for coordination of actuator controller modules, sensor interfaces, and data relay to a computer located on the surface. Communication between the 8-bit core and the surface computer is handled through an Ethernet connection.

\subsection{Software System}

Computation for Ferret is distributed among the several processors located internal and external to the device. Within Ferret, JR Kerr motor controllers drive the PTU actuators; a laser controller issues averaged distance and orientation measurements; and sensor data is routed to interface ports. All these controller and IO devices communicate with the Rabbit core over serial ports and each device is poled for incoming data. If a message is received, the data is extracted and utilized for mechanism control and relayed to the command station.

Above low-level data flow and controller communication, procedures are defined that examine laser orientation and distance measurements to manipulate angular velocity. In scanning mode, Ferret moves the within specified angular bounds (defined from the command station) by monitoring the pan and tilt angular positions. Encountering a pan bound switches the direction of rotation and detecting a tilt bound terminates the scan. Furthermore, the speed of rotation is altered in proportion to the current range measurement to maintain consistent arc lengths between range readings.

On the surface, a computer command station interacts with Ferret over a TCP/IP interface. This computer contains a user control interface (UCI) that allows velocities, accelerations, angular bounds, angle positions, and scan parameters to be set by an operator. Visual indicators in the form of dials and status lights provide sensor feedback so that the surface team can gage Ferret's state throughout operation. The UCI also handles logging, provides data visualization, and allows the user to directly manipulate the PTU for laser and camera direction.

Software that performs post analysis of the data is handled offline and will be discussed in the results section of this paper.

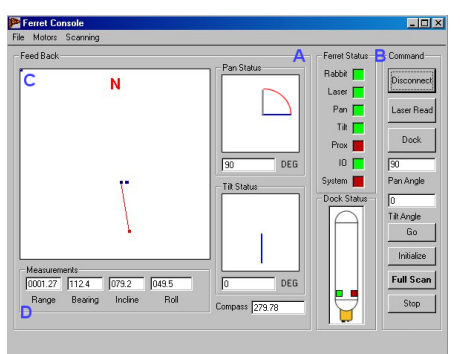

Figure 3: UCI. (A) Ferret status. (B) User commands. (C) Measurement displays. (D) Laser output. 


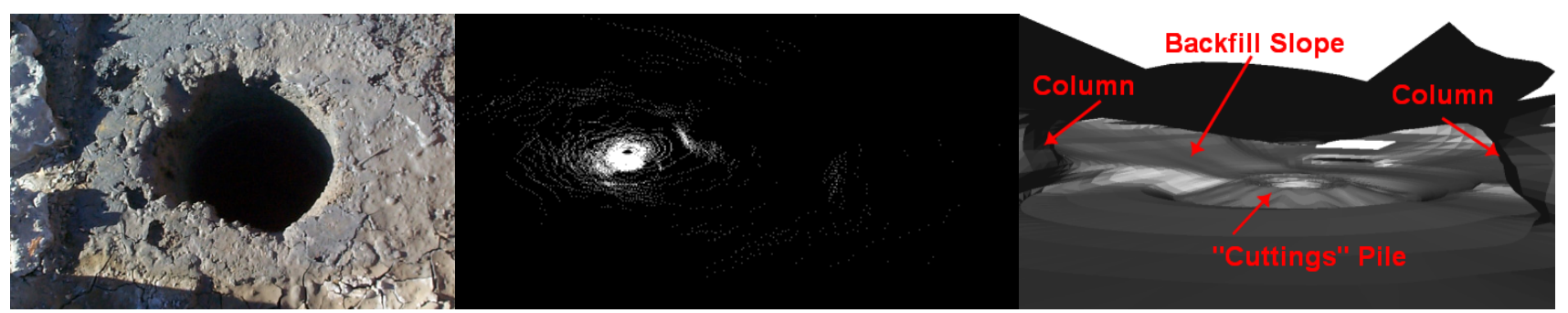

Figure 4: Views of Borehole 1. From left to right: Photograph of borehole with moist clay around edge; 3D pointcloud of all range measurements; mesh view from inside of mine.
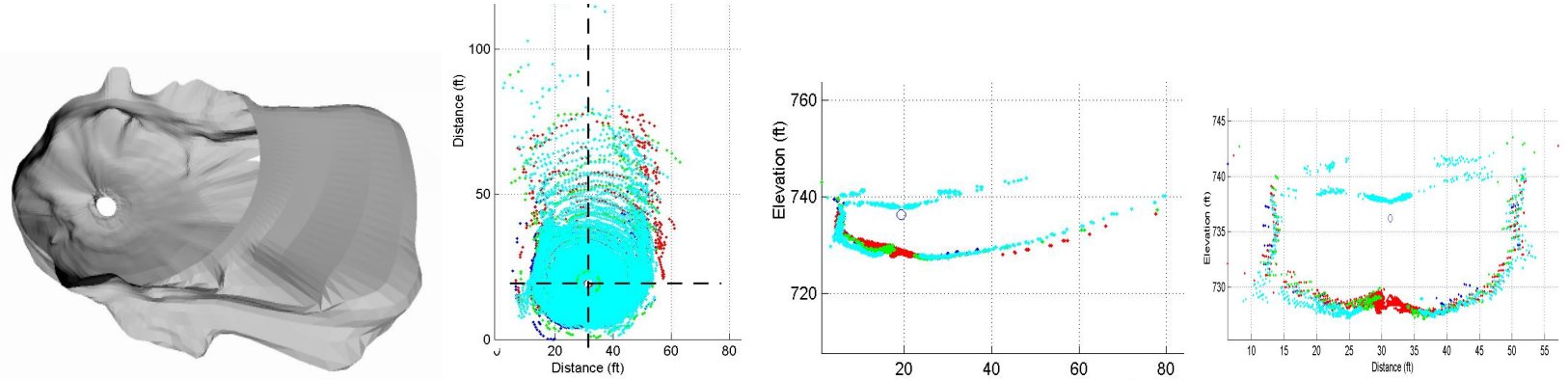

Figure 5: Domeout Scan. From left to right: 3D model; XY projection plot; YZ slice about dotted vertical line in XY plot; XZ slice about dotted horizontal line in XY plot.

\section{Case Study Review}

Over a three-day period in January 2003, Ferret was deployed into an abandoned limestone mine beneath Kansas City for void assessment. The operation was conducted at the request of the landowner who sought to permit overtop a partially backfilled limestone mine. Prior to Ferret, the primary means of gathering information was borehole logs and borehole cameras; however, these methods proved inadequate for determining void extent and unreliable for making decisions on structural integrity.

\subsection{Operational Overview}

Each day of the operation, Ferret scanned a separate section of the mine from one of three borehole vantages. These boreholes were approximately $500 \mathrm{ft}$. spaced from one another with an average depth of $200 \mathrm{ft}$. Scan time averaged 3.5 hours acquiring approximately 38,000 range measurements (a limit imposed by the laser controller over that time frame). In addition, site engineers actively monitored Ferret scans and video to supply real-time feedback and augment data findings.

Environmental conditions were rugged and challenged the functionality of Ferret. For example, the surface temperature was $15^{\circ} \mathrm{F}$ while mine temperature was approximately $50^{\circ} \mathrm{F}$ so that high levels of humidity led to large amounts of water vapor. As a result, dense steam columns emanated from the boreholes and instantly fogged camera and laser lenses upon immersion. This thick cloud of water vapor also moistened the soil along the perimeter of the borehole forming a viscous mud that adhered to the hull, collected in gears, and flooded all openings. Nevertheless, Ferret performed the scans and acquired void data undaunted by conditions of the borehole.

\subsection{Data Analysis}

Upon data acquisition, rigorous analysis was performed offline. Figure 4 shows the point-cloud and mesh created from one of the three scans. In this form, range data permitted a unique 3D geometric visualization that allowed landmark identification of limestone mine features. In this particular scan, a "cuttings" pile (a collection of soil formed during the borehole reaming process), mine columns, and a backfill slope can be seen. These features provided insight on borehole location within the mine and assisted engineers in understanding the work preformed in the previous backfill operation.

Slices of this 3D data permitted dimensional analysis of the voids. Figure 5 shows plots taken from a domeout scan. The cavity size indicated no previous backfill and small pockets of long-range measurements suggested expansion into other sections of the mine. Due to its state, multiple scans were taken at varying elevations. 


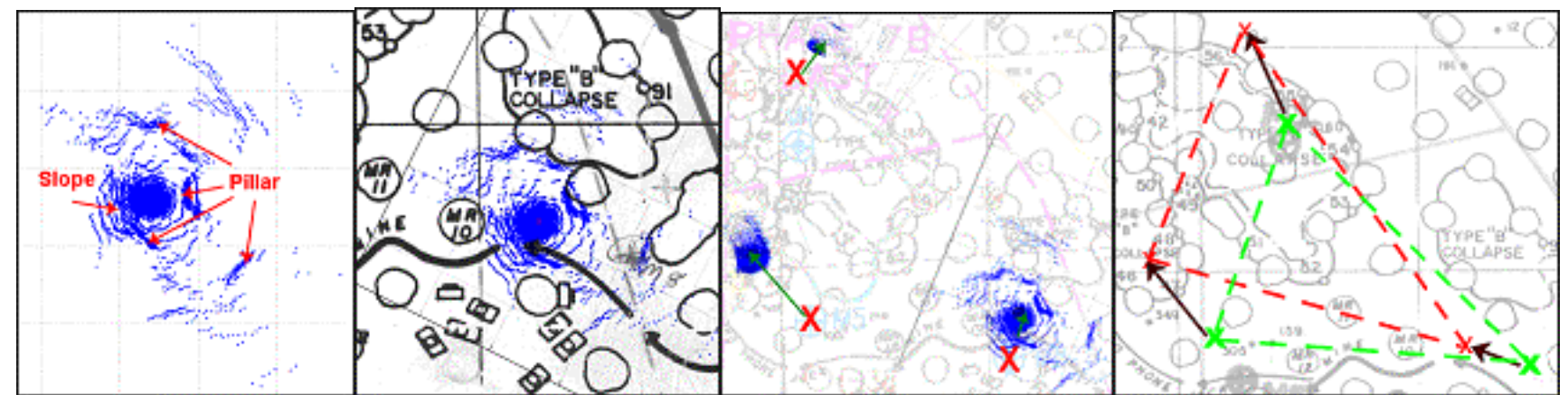

Figure 6: Mine map analysis. From left to right: projection plot of borehole 1; projection plot overlaid on mine map in determined position; all three projection plots placed in same map; calculated warp (green X's mark estimated borehole placement prior to Ferret and red X's mark determined positions).

By varying the laser's vantage, individual scans were geometrically accurate only in local coordinate frames. Motion of the sensor between scans caused the scan sequence to be misaligned. To correct for this misalignment, these data sets were registered in a common coordinate system using an algorithm for simultaneous registration of multiple $3 \mathrm{D}$ data sets. For this purpose, we utilized an implementation of Neugebauer's algorithm [10] (essentially a multi-view extension of the well-known iterative closest point algorithm [11]) to facilitate registration. The algorithm repeatedly adjusts the transforms between scans, minimizing the distance between the surfaces in regions where two scans overlap. Once the scans are aligned in the same coordinate system, the combined data provides a denser and more complete model of the void (Figure 7).

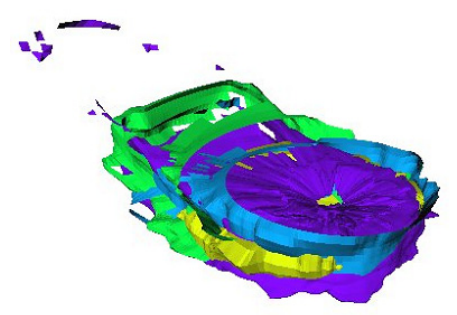

Figure 7: Registration of multiple 3D laser scans.

For mine map correction, a combination of several image-processing techniques and constrained searching methods were used to identify scan locations on the mine map. To start, Euclidean 3D data was projected into the $\mathrm{XY}$ plane to match the map space and form a template that governed the search for optimal placement. These plots provided keying features where high point accumulation signified edges of objects like columns and walls and sparse accumulation indicated open space (periodic measurements from the ceiling and floor).
Utilizing template-matching techniques [12], column edges and solid landmarks on the mine map were correlated with high-density point clusters. To determine an optimal alignment, the search sought to

1. Minimize the number of points lying inside of solid structures

2. Minimize the number of rays from the laser origin to range points that intersected solid structures

3. Maximize the number of points that reside in close proximity to the border of solid structures

In addition, prior compass readings, estimates of borehole locations, and identified features from the $3 \mathrm{D}$ models were assimilated to reduce the search space. Figure 6 shows the projection plot and its correlated position overlaid onto the mine map.

To conclude mine map analysis, the coordinate frame of the map was registered to surface (or global) coordinates. Using the global position of each borehole's surface-side location (obtained from a survey team and GPS positioning), the corresponding mine map was warped into place (Figure 6).

\section{$5 \quad$ Summary and Conclusions}

In summary, the full operation (comprised of three boreholes) consumed three days of fieldwork and three weeks of analysis. Hole 1 was situated over a partially backfilled portion of the mine on the southeastern side of the site. The gap height was approximately $3 \mathrm{ft}$. and range measurements extended out to $90 \mathrm{ft}$. Hole 2 was located in a domeout in the western section of the site with no previous backfill. The domeout was roughly 60 $\mathrm{ft}$. wide, $95 \mathrm{ft}$. long, and $16 \mathrm{ft}$ tall. Hole 3 was in the 
northern section of the site with no perceivable backfill. Due to the unregistered mine map, however, over $50 \%$ of the scan area in Hole 3 was blocked by an adjacent mine pillar. Furthermore, the cuttings pile accumulated almost to the ceiling and created a narrow band of viewing space for scanning. Therefore, the scan was only able to determine cavity extent.

The data and analysis generated by Ferret resulted in a decision to proceed with a second backfill operation. In utilizing Ferret, the number of boreholes required to make this assessment was reduced by $40 \%$ according to site engineers. Instead of inference from camera imagery, quantitative data was available to declare a possible subsidence threat. Currently, arrangements are in progress to recall Ferret to the site to assist during the backfill process.

From this experience, several strengths and weaknesses in the functionality of Ferret were discovered that merit mentioning in this paper. First, the success of boreholedeployable scanning devices at acquiring cavity information is highly dependent upon the location of the boreholes. In this study, both hole 1 and hole 2 provided advantageous locations to view the mine. Hole 3, however, is an example case where poor locality (near solid objects) severely limited the amount of obtainable range information.

Another important issue is device size. In its current configuration, Ferret can access 12 in. diameter boreholes without difficulty. Holes of this size reduce the economical advantage that Ferret employs. A size reduction that permits deployment in 6 in. diameter boreholes would greatly improve the costs for drilling access holes; however, laser equipment of this form factor with the robustness, reliability, and price of the previous Ferret is difficult to fabricate.

In conclusion, the Ferret is a field-operational robotic device that is available for immediate use in subterranean mapping. It provides a remote, underground presence to acquire highly accurate 3D models in locations where hazardous conditions prohibit human access. In this Kansas City case study, Ferret was shown to be a valuable component in realizing, measuring, and mapping abandoned limestone mine sections and domeouts for structural analysis and remediation. Furthermore, this study suggests the flexibility of Ferret technology to other application domains such as verification of abandoned coalmines and situational analysis of subterranean hazardous waste disposals.
The land owning company and engineering firm in Kansas City who learned of our technology developments and had the insight to implement us into their plans. Workhorse Technology, LLC, the company located in Homestead, PA dedicated to subterranean mapping that funded and supported the development of Ferret and mine mapping initiatives at Carnegie Mellon University. The Kansas City fieldwork was conducted under contract through Workhorse. Meritex Enterprises that provided development and testing opportunities for Ferret at their Gateway Commerce Center limestone storage facility in Wampum, PA.

\section{$6 \quad$ References}

1. M. Ross and M. Roth. "All Nine Alive: The Story of the Quecreek Mine Rescue.” Pittsburgh Post-Gazette 04 August 2002.

2. Beck, Barry F. and Herring, J.Gayle (eds): Geotechnical and environmental applications of karst geology and hydrology - Proceedings of the 8th multidisciplinary conference on sinkholes \& karst, Louisville, Kentucky, USA, 1-4 April 2001

3. MSHA Public Safety Campaign Stresses That Mines and Minors Don't Mix. MSHA Press Release, U.S. Dept. of Labor, 18 April 2001.

4. P. Burton, A. Kreeger, B. Shefchik. "The Use of Flyash in Mine Stabilization." TechBriefs, 2002 vol. 2.

5. "Ground Penetrating Radar." U.S. Environmental Protection Agency Field Analytic Technologies Encyclopedia, http://fate.clu-in.org/gpr.asp?techtypeid=41 (current May 2003).

6. G. Senechal, F. Hollender, D. Russet. "Near Surface Characterization of a Limestone Site Using Borehole and Surface Geophysics." Geophysical Research Abstracts Vol. 5.

7. "Microgravity Surveying," Keele University Applied \& Environmental Geophysics Research Group, http://www.esci.keele.ac.uk/geophysics/Research/microgra vity surveying.html (current May 2003)

8. Mark Products, Inc. GeoVISION ${ }^{\mathrm{TM}}$ Video Borehole Inspection System. Online documentation: www.marksproducts.com, December 2002.

9. B. Wells. "The Latest Developments in Laser Profiling, Borehole Deviation, and Laser Enhanced Videometry." Measurement Devices Ltd., 1999.

10. P. Neugebauer. "Reconstruction of Real-World Objects Via Simultaneous Registration and Robust Combination of Multiple Range Images," International Journal of Shape Modeling, 1997. Vol. 3, pp 71-90.

11. P. Besl and N. McKay. "A Method of Registration of $\{3 \mathrm{D}\}$ Shapes," jPAMI, Feb. 1992. Vol. 14, pp 239-256.

12. D. Forsyth and J. Ponce. Computer Vision: A Modern Approach, Prentice Hall, Ed. 1, 2002.

\section{Acknowledgements}

